\title{
Synthesis of 4-Amino-4,5-dihydro-1H-1,2,4-triazole-5-ones and their Isatin-3-imine Derivatives
}

\section{Bahittin Kahveci}

Department of Chemistry, Rize Faculty of Arts and Science, Karadeniz Technical University, 53100, Rize, Turkey.

* Author to whom correspondence should be addressed; e-mail: bahittin@yahoo.com

Received: 12 July 2004 / Accepted: 1 September 2004 / Published: 28 February 2005

\begin{abstract}
Iminoester hydrochlorides 1 have been synthesized. These compounds were then converted into ester ethoxycarbonyl hydrazones $\mathbf{2}$, from which in turn a new series of substituted 4-amino-4,5-dihydro-1H-1,2,4-triazole-5-ones, 3, was then prepared. Finally a set of isatin imine derivatives 4 was obtained from the reaction of compounds 3 with isatin. The structures of all the new synthesized compounds were confirmed by elemental analyses, IR, ${ }^{1} \mathrm{H}-\mathrm{NMR}$ and ${ }^{13} \mathrm{C}$-NMR spectra.
\end{abstract}

Keywords: Iminoesters; ethoxycarbonyl hydrazones; 4-amino-1,2,4-triazole-5-ones; isatin

\section{Introduction}

The synthesis and antibacterial activity of some 4-amino-4,5-dihydro-1H-1,2,4-triazoles and their derivatives have been reported in the literature [1-5]. A number of studies involving the antibacterial and antitumor activity of some 4-amino-4,5-dihydro-1H-1,2,4-triazole-5-ones and their derivatives have also been published recently [6-14]. There are also many studies on isatin (1H-indole-2,3-dione) in the literature. The synthetic versatility of isatin has led to the extensive use of this compound in organic synthesis. This has stemmed in great part from the interest in the biological and pharmaceutical properties of its derivatives [15-24]. Although reactions of isatin with many amino compounds have been investigated, it appears that its reaction with the $\mathrm{N}$-aminotriazole-5-ones have not been studied before. Consequently, some new 4-amino-4,5-dihydro-1H-1,2,4-triazole-5-ones have now been synthesized and their reactions with isatin investigated. 


\section{Results and Discussion}

The new derivatives were prepared following the reaction sequences depicted in Scheme 1 . Iminoester starting materials 1a-d were prepared by passing $\mathrm{HCl}$ gas through solutions of 2-chlorobenzyl cyanide, 3-chlorobenzyl cyanide, 2-methylbenzyl cyanide or 3-methylbenzyl cyanide and absolute ethanol, followed by precipitation with ether.

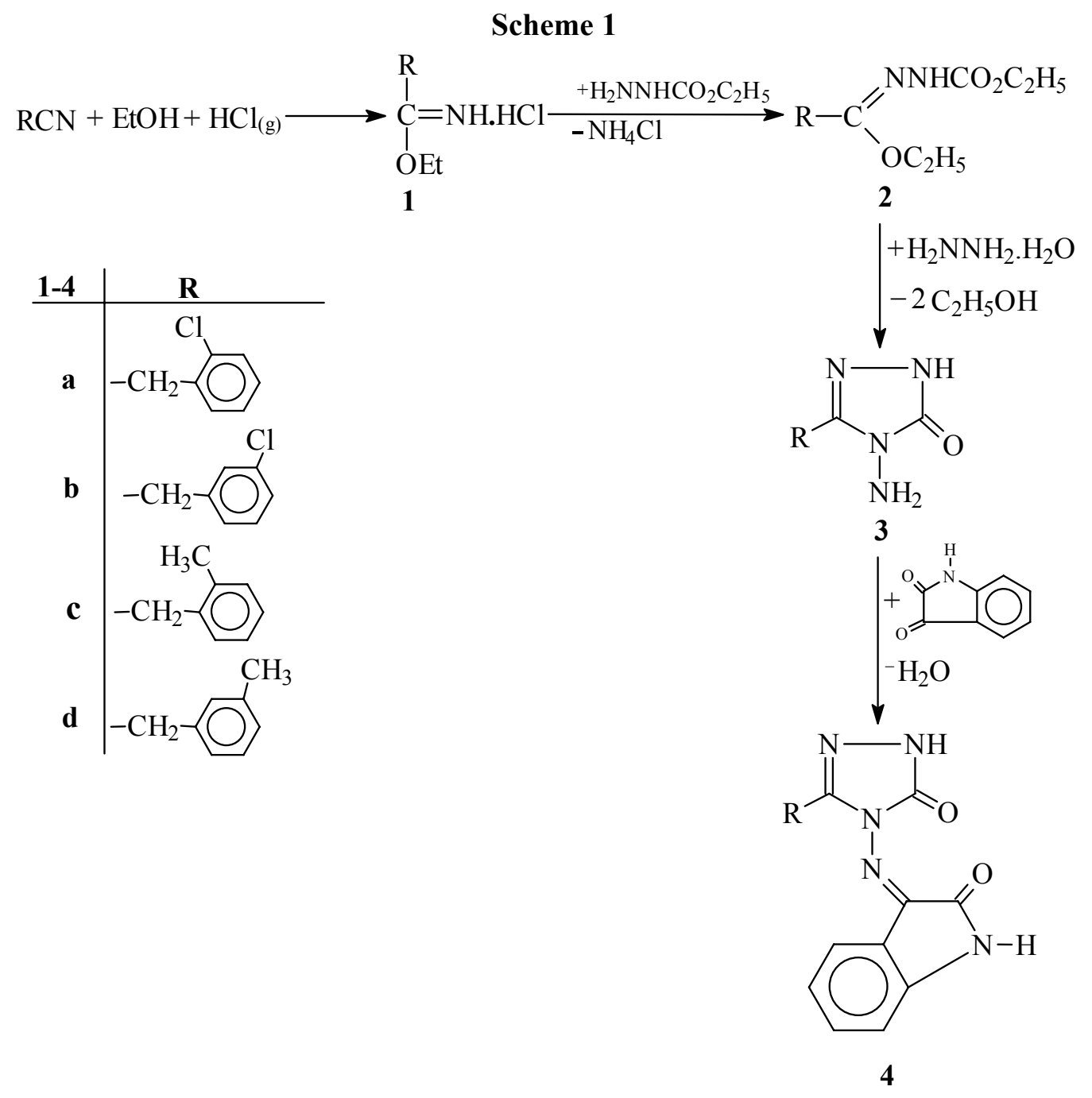

In the IR spectra of compounds 1a-d the characteristic $\mathrm{NH}_{2}{ }^{+}$absorption bands appeared at 2980, 2920 and $810 \mathrm{~cm}^{-1}$ and the $\mathrm{C}=\mathrm{N}$ band at $1640 \mathrm{~cm}^{-1}$, approximately. Reaction of the iminoesters with ethyl carbazate yielded the ethoxycarbonyl hydrazones 2a-d. The IR spectra of these compounds show the $\mathrm{C}=\mathrm{O}$ band at $1740 \mathrm{~cm}^{-1}$ and the $\mathrm{C}=\mathrm{N}$ band at $1600 \mathrm{~cm}^{-1}$, approximately. The obtained compounds 2a-d were then refluxed with a solution of hydrazine hydrate in water to afford the compounds 3a-d. The $\mathrm{NH}_{2}, \mathrm{C}=\mathrm{O}$ and $\mathrm{C}=\mathrm{N}$ bands observed in the IR spectra of the latter compounds match the peaks expected for these structures. In addition to this, observation of $\mathrm{NH}_{2}, \mathrm{NH}$ and aromatic protons, as well as the disappearance of $\mathrm{CH}_{2} \mathrm{CH}_{3}$ signals in the ${ }^{1} \mathrm{H}-\mathrm{NMR}$ spectra and the triazole $\mathrm{C}_{5}$ peak at near $\delta 154$ ppm and the triazole $\mathrm{C}_{3}$ peak at near $\delta 147 \mathrm{ppm}$ in the ${ }^{13} \mathrm{C}$-NMR spectra all support this conclusion. Finally, in the reaction of compounds 3a-d with isatin we obtained the expected isatin-3-imines 4 . The 
structures of the isatin-3-imines were verified by the presence in the IR spectra of two different $\mathrm{C}=\mathrm{O}$ absorbtion bands at around $1700 \mathrm{~cm}^{-1}$ and $\mathrm{C}=\mathrm{N}$ bands near $1690 \mathrm{~cm}^{-1}$. In addition to this, the appearance of signals corresponding to two different $\mathrm{NH}$ protons and the disappearance of the $\mathrm{NH}_{2}$ protons in the ${ }^{1} \mathrm{H}$-NMR spectra support the proposed structures. The ${ }^{13} \mathrm{C}$-NMR spectra also support the expected structures.

\section{Conclusions}

New compounds 3a-d, which can be used to prepare many new compounds, have been synthesized. Some of the new derivatives might be important biologically active agents. The compounds 4a-d are also potential biologically active agents and their medical research applications should be investigated.

\section{Experimental}

\section{General}

Melting points were determined in open capillary tubes on a Büchi oil heated melting point apparatus and are uncorrected. The IR spectra were recorded for $\mathrm{KBr}$ pellets on Perkin-Elmer 1600 FTIR spectrophotometer. ${ }^{1} \mathrm{H}-\mathrm{NMR}$ and ${ }^{13} \mathrm{C}$-MR spectra were recorded on a Varian $200 \mathrm{~A}$ spectrometer (solvent DMSO-d6, TMS as internal standard). Elemental analyses were performed on a Carlo Erba $1106 \mathrm{CHN}$ analyzer. Starting materials were obtained from Fluka or Aldrich.

\section{Synthesis of Iminoester Hydrochlorides 1a-d.}

These were synthesized using the reported method [25]. To an ice-cooled solution of the appropriate nitrile ( 1 mole) in absolute alcohol ( 1.1 moles), dry hydrogen chloride was added until 1.1 moles had been absorbed. The resulting solution was then allowed to stand at $0{ }^{\circ} \mathrm{C}$ in the refrigerator for 12 hours, after which cold absolute ether was added and the obtained crystals were filtered off immediately, washed with cold absolute ether and dried in a dessicator. The following compounds were thus prepared:

Ethyl imido-o-chlorophenylacetate hydrochloride (1a): m.p. $95-96{ }^{\circ} \mathrm{C}$; IR $\bar{v}\left(\mathrm{~cm}^{-1}\right): 2982,2878,812$ $\left(\mathrm{NH}_{2}{ }^{+}\right), 1624(\mathrm{C}=\mathrm{N}), 753$ (1,2-disubstituted benzene ring).

Ethyl imido-m-chlorophenylacetate hydrochloride (1b): m.p. 82-84 ${ }^{\circ} \mathrm{C} ; \mathrm{IR} \bar{v}\left(\mathrm{~cm}^{-1}\right): 2989,2939,823$ $\left(\mathrm{NH}_{2}^{+}\right), 1649(\mathrm{C}=\mathrm{N}), 868,791,684$ (1,3-disubstituted benzene ring).

Ethyl imido-o-methylphenylacetate hydrochloride (1c): m.p. $80-81{ }^{\circ} \mathrm{C}$; IR $\bar{v}\left(\mathrm{~cm}^{-1}\right): 2981,2928,823$ $\left(\mathrm{NH}_{2}{ }^{+}\right), 1648(\mathrm{C}=\mathrm{N}), 747$ (1,2-disubstituted benzene ring).

Ethyl imido-m-methylphenylacetate hydrochloride (1d): m.p. 113-114 ${ }^{\circ} \mathrm{C}$; IR $\bar{v}\left(\mathrm{~cm}^{-1}\right): 2994,2862$, $811\left(\mathrm{NH}_{2}{ }^{+}\right), 1653(\mathrm{C}=\mathrm{N}), 867,768,714$ (1,3-disubstituted benzene ring). 
General Procedure for Preparation of Ethyl Substituted Formate Ethoxycarbonyl hydrazones 2a-d.

In a stoppered flask equipped with a magnetic stirrer, the corresponding ethyl imidocarboxylate hydrochloride (1a-d, $0.01 \mathrm{~mol})$ was dissolved in absolute ethanol $(50 \mathrm{~mL})$ with ice-bath cooling and ethyl carbazate $(0.01 \mathrm{~mol})$ dissolved in absolute ethanol $(20 \mathrm{~mL})$ was then added to this solution. After stirring for $6 \mathrm{hr}$ in ice-bath, the mixture was filtered to remove the ammonium chloride which separated from the solution and the filtrate was evaporated at $30-35^{\circ} \mathrm{C}$ under reduced pressure. The solid residue, after drying in a dessicator, was recrystallized from petroleum ether to yield compounds 2a-d.

Ethyl o-chlorophenylacetate ethoxycarbonyl hydrazone (2a): Prepared from 1a, yield 78\%; m.p. 54-55 ${ }^{\circ} \mathrm{C}$; IR $\bar{v}\left(\mathrm{~cm}^{-1}\right): 3274(\mathrm{~N}-\mathrm{H}), 1721(\mathrm{C}=\mathrm{O}), 1648(\mathrm{C}=\mathrm{N}), 751$ (1,2-disubstituted benzene ring); ${ }^{1} \mathrm{H}-$ NMR, $\delta(\mathrm{ppm}): 1.18\left(\mathrm{t}, 3 \mathrm{H}, \mathrm{CH}_{3}\right), 1.28\left(\mathrm{t}, 3 \mathrm{H}, \mathrm{CH}_{3}\right), 3.71\left(\mathrm{~s}, 2 \mathrm{H}, \mathrm{CH}_{2}\right), 3.96\left(\mathrm{q}, 2 \mathrm{H}, \mathrm{CH}_{2}\right), 4.24(\mathrm{q}, 2 \mathrm{H}$, $\mathrm{CH}_{2}$ ), 6.58-7.24 (m, 4H, Ar-H), 8.18 (s, $1 \mathrm{H}, \mathrm{NH}$ ); Calcd. (\%) for $\mathrm{C}_{13} \mathrm{H}_{17} \mathrm{~N}_{2} \mathrm{O}_{3} \mathrm{Cl}$ (285): C, 54.78; $\mathrm{H}$, $6.02 ; \mathrm{N}, 9.84$; found (\%): C, 54.92; H, 6.01, N, 10.00 .

Ethyl m-chlorophenylacetate ethoxycarbonyl hydrazone (2b): Prepared from 1b, 74\%; m.p. 47-48 ${ }^{\circ} \mathrm{C}$; IR $\bar{v}\left(\mathrm{~cm}^{-1}\right)$ : $3247(\mathrm{~N}-\mathrm{H}), 1709(\mathrm{C}=\mathrm{O}), 1648(\mathrm{C}=\mathrm{N}), 863,770,683$ (1,3-disubstituted benzene ring); ${ }^{1} \mathrm{H}-\mathrm{NMR}, \delta(\mathrm{ppm}): 1.19$ (t, 3H, $\left.\mathrm{CH}_{3}\right), 1.24\left(\mathrm{t}, 3 \mathrm{H}, \mathrm{CH}_{3}\right), 3.64\left(\mathrm{~s}, 2 \mathrm{H}, \mathrm{CH}_{2}\right), 3.91\left(\mathrm{q}, 2 \mathrm{H}, \mathrm{CH}_{2}\right), 4.11$ (q, $\left.2 \mathrm{H}, \mathrm{CH}_{2}\right), 6.80-7.11(\mathrm{~m}, 4 \mathrm{H}, \mathrm{Ar}-\mathrm{H}), 8.23$ (s, $1 \mathrm{H}, \mathrm{NH}$ ); Calcd. (\%) for $\mathrm{C}_{13} \mathrm{H}_{17} \mathrm{~N}_{2} \mathrm{O}_{3} \mathrm{Cl}$ (285): C, 54.78; H, 6.02; N, 9.84; found (\%): C, 54.48; H, 6.18, N, 10.27.

Ethyl o-methylphenylacetate ethoxycarbonyl hydrazone (2c): Prepared from 1c, yield 80\%; m.p. 66-67 ${ }^{\circ} \mathrm{C}$; IR $\bar{v}\left(\mathrm{~cm}^{-1}\right): 3207(\mathrm{~N}-\mathrm{H}), 1702(\mathrm{C}=\mathrm{O}), 1657(\mathrm{C}=\mathrm{N}), 747$ (1,2-disubstituted benzene ring); 'H-NMR, $\delta$ (ppm): $1.20\left(\mathrm{t}, 3 \mathrm{H}, \mathrm{CH}_{3}\right), 1.32\left(\mathrm{t}, 3 \mathrm{H}, \mathrm{CH}_{3}\right), 2.30\left(\mathrm{~s}, 3 \mathrm{H}, \mathrm{CH}_{3}\right), 3.64\left(\mathrm{~s}, 2 \mathrm{H}, \mathrm{CH}_{2}\right), 4.00\left(\mathrm{q}, 2 \mathrm{H}, \mathrm{CH}_{2}\right)$, 4.24 (q, 2H, $\left.\mathrm{CH}_{2}\right), 6.96-7.30$ (m, 4H, Ar-H); Calcd. (\%) for $\mathrm{C}_{14} \mathrm{H}_{20} \mathrm{~N}_{2} \mathrm{O}_{3}$ (264): $\mathrm{C}, 63.56 ; \mathrm{H}, 7.63 ; \mathrm{N}$, 10.60; found (\%): C, 63.68; H, 7.89, N, 10.86 .

Ethyl m-methylphenylacetate ethoxycarbonyl hydrazone (2d): Prepared from 1d, yield 86\%; m.p. 59$60{ }^{\circ} \mathrm{C}$; IR $\bar{v}\left(\mathrm{~cm}^{-1}\right): 3257(\mathrm{~N}-\mathrm{H}), 1710(\mathrm{C}=\mathrm{O}), 1648(\mathrm{C}=\mathrm{N}), 873,755,694$ (1,3-disubstituted benzene ring); ${ }^{1} \mathrm{H}-\mathrm{NMR}, \delta(\mathrm{ppm}): 1.21\left(\mathrm{t}, 3 \mathrm{H}, \mathrm{CH}_{3}\right), 1.28\left(\mathrm{t}, 3 \mathrm{H}, \mathrm{CH}_{3}\right), 2.28\left(\mathrm{~s}, 3 \mathrm{H}, \mathrm{CH}_{3}\right), 3.60\left(\mathrm{~s}, 2 \mathrm{H}, \mathrm{CH}_{2}\right)$, 4.08 (q, 2H, $\left.\mathrm{CH}_{2}\right), 4.20$ (q, 2H, $\left.\mathrm{CH}_{2}\right), 6.96-7.54(\mathrm{~m}, 4 \mathrm{H}, \mathrm{Ar}-\mathrm{H}), 8.46$ (s, 1H, NH); Calcd. (\%) for $\mathrm{C}_{14} \mathrm{H}_{20} \mathrm{~N}_{2} \mathrm{O}_{3}(264)$ : C, 63.56; H, 7.63; N, 10.60; found (\%): C, 63.65; H, 7.89, N, 10.58 .

General Procedure for the Preparation of 3-Substituted-4-amino-4,5-dihydro-1H-1,2,4-triazole-5ones 3a-d.

Compound $2(0.01 \mathrm{~mol})$ was refluxed for $5 \mathrm{hr}$ with a solution of hydrazine hydrate $(1.25 \mathrm{~mL})$ in water $(60 \mathrm{~mL})$. The solution was crystallized by cooling to obtain the crude product (3a-d). The solid material thus obtained was filtered off and recrystallized from ethanol. 
3-o-Chlorobenzyl-4-amino-4,5-dihydro-1H-1,2,4-triazole-5-one (3a): Prepared from 2a, yield 88\%; m.p. $164-165^{\circ} \mathrm{C}$; IR $\bar{v}\left(\mathrm{~cm}^{-1}\right)$ : 3338, $3217\left(\mathrm{NH}_{2}, \mathrm{NH}\right), 1720(\mathrm{C}=\mathrm{O}), 1633(\mathrm{C}=\mathrm{N}), 749$ (1,2-disubstituted benzene ring); ${ }^{1} \mathrm{H}-\mathrm{NMR}, \delta(\mathrm{ppm}): 4.10\left(\mathrm{~s}, 2 \mathrm{H}, \mathrm{CH}_{2}\right), 5.28$ (s, 2H, $\left.\mathrm{NH}_{2}\right), 7.25$ (s, 4H, Ar-H), 11.90 (s, $1 \mathrm{H}, \mathrm{NH}) ;{ }^{13} \mathrm{C}-\mathrm{NMR}, \delta$ (ppm): 28.80, 124.63, 126.84, 130.13, 132.86, 136.90, 138.63, 145.10, 153.14;. Calcd. (\%) for $\mathrm{C}_{9} \mathrm{H}_{9} \mathrm{~N}_{4} \mathrm{OCl}(225)$ : C, 48.08; H, 4.04; N, 24.94; found (\%): C, 48.40; H, 4.54, N, 24.68.

3-m-Chlorobenzyl-4-amino-4,5-dihydro-1H-1,2,4-triazole-5-one (3b):Prepared from 2b, yield 83\%; m.p. $171-172{ }^{\circ} \mathrm{C}$; IR $\bar{v}\left(\mathrm{~cm}^{-1}\right)$ : 3324, $3224\left(\mathrm{NH}_{2}, \mathrm{NH}\right), 1730(\mathrm{C}=\mathrm{O}), 1638(\mathrm{C}=\mathrm{N}), 864,793,719(1,3-$ disubstituted benzene ring); ${ }^{1} \mathrm{H}-\mathrm{NMR}, \delta(\mathrm{ppm}): 4.00\left(\mathrm{~s}, 2 \mathrm{H}, \mathrm{CH}_{2}\right), 5.18\left(\mathrm{~s}, 2 \mathrm{H}, \mathrm{NH}_{2}\right), 7.16$ (s, 4H, Ar$\mathrm{H}), 11.10(\mathrm{~s}, 1 \mathrm{H}, \mathrm{NH}) ;{ }^{13} \mathrm{C}-\mathrm{NMR}, \delta(\mathrm{ppm}): 29.62,125.54,127.84,131.14,133.62,136.95,137.44$, 146.93, 154.11; Calcd. (\%) for $\mathrm{C}_{9} \mathrm{H}_{9} \mathrm{~N}_{4} \mathrm{OCl}$ (225): C, 48.08; H, 4.04; N, 24.94; found (\%): C, 48.34; $\mathrm{H}$, $4.63, \mathrm{~N}, 24.85$.

3-o-Methylbenzyl-4-amino-4,5-dihydro-1H-1,2,4-triazole-5-one (3c): Prepared from 2c, yield 91\%; m.p. 190-191 ${ }^{\circ} \mathrm{C}$; IR $\bar{v}\left(\mathrm{~cm}^{-1}\right)$ : 3327, $3221\left(\mathrm{NH}_{2}, \mathrm{NH}\right), 1725(\mathrm{C}=\mathrm{O}), 1635(\mathrm{C}=\mathrm{N}), 736(1,2$-disubstituted benzene ring); ${ }^{1} \mathrm{H}$ NMR, $\delta$ (ppm): $2.38\left(\mathrm{~s}, 3 \mathrm{H}, \mathrm{CH}_{3}\right), 4.10\left(\mathrm{~s}, 2 \mathrm{H}, \mathrm{CH}_{2}\right), 5.28\left(\mathrm{~s}, 2 \mathrm{H}, \mathrm{NH}_{2}\right), 7.32(\mathrm{~s}, 4 \mathrm{H}$, $\mathrm{Ar}-\mathrm{H}), 11.60$ (s, 1H, NH); ${ }^{13} \mathrm{C}-\mathrm{NMR}, \delta(\mathrm{ppm}): 20.12,29.76,124.86,125.18,129.26,130.18,134.10$, 135.19, 146.13, 153.81; Calcd. (\%) for $\mathrm{C}_{10} \mathrm{H}_{12} \mathrm{~N}_{4} \mathrm{O}$ (204): C, 58.76; H, 5.92; N, 27.42; found (\%): C, 58.86; H, 6.04, N, 27.31.

3-m-Methylbenzyl-4-amino-4,5-dihydro-1H-1,2,4-triazole-5-one (3d): Prepared from 2d, yield 87\%; m.p.156-157 ${ }^{\circ} \mathrm{C}$; IR $\bar{v}\left(\mathrm{~cm}^{-1}\right)$ : 3322, $3222\left(\mathrm{NH}_{2}, \mathrm{NH}\right), 1731(\mathrm{C}=\mathrm{O}), 1642(\mathrm{C}=\mathrm{N}), 822,751,705(1,3-$ disubstituted benzene ring); ${ }^{1} \mathrm{H}-\mathrm{NMR}, \delta(\mathrm{ppm}): 2.38\left(\mathrm{~s}, 3 \mathrm{H}, \mathrm{CH}_{3}\right), 4.16\left(\mathrm{~s}, 2 \mathrm{H}, \mathrm{CH}_{2}\right), 5.24(\mathrm{~s}, 2 \mathrm{H}$, $\mathrm{NH}_{2}$ ), 7.24 (s, 4H, Ar-H), $11.46(\mathrm{~s}, 1 \mathrm{H}, \mathrm{NH}) ;{ }^{13} \mathrm{C}-\mathrm{NMR}, \delta(\mathrm{ppm}): 19.08,28.12,125.70,126.56$, 129.01, 129.78, 134.27, 136.17, 147.28, 154.00; Calcd. (\%) for $\mathrm{C}_{10} \mathrm{H}_{12} \mathrm{~N}_{4} \mathrm{O}$ (204): C, 58.76; H, 5.92; N, 27.42; found (\%): C, 58.69; H, 6.12, N, 27.96.

General Procedure for the Preparation of Isatin-3-imines 4a-d.

Equimolar quantities $(0.01 \mathrm{~mol})$ of isatin and the corresponding amino compound 3a-d were dissolved in warm ethanol $(40 \mathrm{~mL})$ containing glacial acetic acid $(0.5 \mathrm{~mL})$. The reaction mixture was refluxed for $4 \mathrm{hr}$ and then kept at room temperature overnight. The resulting solid was washed with ethanol, dried and recrystallized from ethanol-chloroform to afford compounds 4a-d.

3-[3'-(4',-o-Chlorobenzyl-4',5'-dihydro-1'H-1',2',4'-triazol-5'-on-4'-yl]-iminoisatin (4a): Prepared from 3a, yield 72\%; m.p. 252-253 ${ }^{\circ} \mathrm{C}$; IR $\bar{v}\left(\mathrm{~cm}^{-1}\right)$ : 3403, $3190(\mathrm{~N}-\mathrm{H}), 1754,1730(\mathrm{C}=\mathrm{O}), 1684,1613$ $(\mathrm{C}=\mathrm{N}), 1463(\mathrm{C}=\mathrm{C}) ;{ }^{1} \mathrm{H}-\mathrm{NMR}, \delta(\mathrm{ppm}): 4.18\left(\mathrm{~s}, 2 \mathrm{H}, \mathrm{CH}_{2}\right), 7.18-7.64(\mathrm{~m}, 8 \mathrm{H}, \mathrm{Ar}-\mathrm{H}), 11.22(\mathrm{~s}, 1 \mathrm{H}, \mathrm{N}-$ $\mathrm{H}), 12.40(\mathrm{~s}, 1 \mathrm{H}, \mathrm{N}-\mathrm{H}) ;{ }^{13} \mathrm{C}-\mathrm{NMR}, \delta$ (ppm): 29.26, 113.42, 115.80, 121.18, 125.50, 126.18, 128.88, $129.85,131.60,133.36,134.81,137.18,144.81,145.83,146.12,156.18,161.86$; Calcd. (\%) for $\mathrm{C}_{17} \mathrm{H}_{12} \mathrm{~N}_{5} \mathrm{O}_{2} \mathrm{Cl}$ (354): C, 67.66; H, 3.42; N, 19.79; found (\%): C, 57.18; H, 3.52, N, 19.64. 
3-[3'-(4' '-m-Chlorobenzyl)-4',5'-dihydro-1'H-1',2',4'-triazol-5'-on-4'-yl]-iminoisatin (4b): Prepared from 3b, yield 68\%; m.p. 266-267 ${ }^{\circ} \mathrm{C}$; IR $\bar{v}(\mathrm{~cm}-1)$ : 3164, $3086(\mathrm{~N}-\mathrm{H}), 1730,1708(\mathrm{C}=\mathrm{O}), 1684,1613$ $(\mathrm{C}=\mathrm{N}), 1464(\mathrm{C}=\mathrm{C}) ;{ }^{1} \mathrm{H}-\mathrm{NMR}, \delta(\mathrm{ppm}): 4.14\left(\mathrm{~s}, 2 \mathrm{H}, \mathrm{CH}_{2}\right), 6.96-7.32(\mathrm{~m}, 6 \mathrm{H}, \mathrm{Ar}-\mathrm{H}), 7.40-7.68(\mathrm{~s}, 2 \mathrm{H}$, Ar-H), 11.28 (s, 1H, N-H), 12.36 (s, 1H, N-H); ${ }^{13} \mathrm{C}-\mathrm{NMR}, \delta$ (ppm): 28.31, 112.41, 116.42, 120.00, $124.81,127.51,128.12,129.24,131.18,132.41,135.40,136.02,145.10,145.91,146.83,157.16$, 162.15; Calcd. (\%) for $\mathrm{C}_{17} \mathrm{H}_{12} \mathrm{~N}_{5} \mathrm{O}_{2} \mathrm{Cl}$ (354): C, 57.66; H, 3.42; N, 19.79; found (\%): C, 57.89; H, 3.48, N, 19.69 .

3-[3'-(4',-o-Methylbenzyl)-4',5'-dihydro-1 'H-1 ',2',4'-triazol-5'-on-4'-yl]-iminoisatin (4c): Prepared from 3c, yield 74\%; m.p. 269-270 ${ }^{\circ} \mathrm{C}$; IR $\bar{v}\left(\mathrm{~cm}^{-1}\right)$ : 3194, $3088(\mathrm{~N}-\mathrm{H}), 1746,1706(\mathrm{C}=\mathrm{O}), 1610,1588$ $(\mathrm{C}=\mathrm{N}), 1467(\mathrm{C}=\mathrm{C}) ;{ }^{1} \mathrm{H}-\mathrm{NMR}, \delta(\mathrm{ppm}): 2.44\left(\mathrm{~s}, 3 \mathrm{H}, \mathrm{CH}_{3}\right), 4.08\left(\mathrm{~s}, 2 \mathrm{H}, \mathrm{CH}_{2}\right), 6.92-7.66(\mathrm{~m}, 8 \mathrm{H}, \mathrm{Ar}-$ $\mathrm{H}), 11.12$ (s, 1H, N-H), 12.54 (s, 1H, N-H); ${ }^{13} \mathrm{C}-\mathrm{NMR}, \delta$ (ppm): 19.10, 29.33, 111.16, 115.73, 122.41, $125.74,127.10,127.90,129.37,130.04,133.33,135.54,136.53,145.70,146.10,148.54,158.04$, 163.31; Calcd. (\%) for $\mathrm{C}_{18} \mathrm{H}_{15} \mathrm{~N}_{5} \mathrm{O}_{2}$ (333): C, 64.80; H, 4.54; N, 21.00; found (\%): C, 64.96; H, 4.66, N, 21.44.

3-[3'-(4',-m-Methylbenzyl)-4',5'-dihydro-1'H-1',2',4'-triazol-5'-on-4'-yl]-iminoisatin (4d): Prepared from 3d, yield $75 \%$; m.p. 272-273 ${ }^{\circ} \mathrm{C}$; IR õ $\left(\mathrm{cm}^{-1}\right)$ : 3177, $3084(\mathrm{~N}-\mathrm{H}), 1748,1707(\mathrm{C}=\mathrm{O}), 1609,1583$ $(\mathrm{C}=\mathrm{N}), 1466(\mathrm{C}=\mathrm{C}) ;{ }^{1} \mathrm{H}-\mathrm{NMR} \delta(\mathrm{ppm}): 2.41(\mathrm{~s}, 3 \mathrm{H}, \mathrm{CH}), 4.12\left(\mathrm{~s}, 2 \mathrm{H}, \mathrm{CH}_{2}\right), 7.00-7.47$ (m, 8H, Ar-H), $11.20(\mathrm{~s}, 1 \mathrm{H}, \mathrm{N}-\mathrm{H}), 12.32$ (s, 1H, N-H); ${ }^{13} \mathrm{C}-\mathrm{NMR}, \delta$ (ppm): 19.04, 28.96, 112.86, 114.61, 121.14, $124.81,127.00,127.50,128.14,131.14,132.15,135.20,135.41,144.62,146.54,147.51,157.60$, 162.94; Calcd. (\%) for $\mathrm{C}_{18} \mathrm{H}_{15} \mathrm{~N}_{5} \mathrm{O}_{2}$ (333): C, 64.80; H, 4.54; N, 21.00; found (\%): $\mathrm{C}, 64.56 ; \mathrm{H}, 4.63$, $\mathrm{N}, 21.21$.

\section{References}

1. Ün, R.; İkizler, A.A. Chim. Acta Turc. 1975, 3, 1-22.

2. İkizler, A.A.; Ün, R. Chim Acta Turc. 1979, 7, 269-290.

3. Milcent, R.; Redeuilh, C. J. Heterocycl. Chem. 1979, 16, 403-407.

4. Milcent, R.; Redeuilh, C. J. Heterocycl. Chem. 1980, 17, 1691-1696.

5. Malbec, F.; Micent, R. Vicart, P. J. Heterocycl. Chem. 1984, 21, 1769-1774.

6. İkizler, A.A.; İkizler, A.; Yıldırım, N. Monatsh. Chem. 1991, 122, 557-563.

7. İkizler, A.; Demirbaş, N.; Demirbaş, A.; İkizler, A.A. Polish. J. Chem. 1996, 70, 1114-1120.

8. Yüksek, H.; Demirbaş, A.; İkizler, A.; Johansson, C.; Çelik, C.; İkizler, A.A. Arzneim.Forsch./Drug Res. 1997, 47(I), 405-409.

9. Serdar, M.; Sivri, E.; Yavuz, E.; Demirbaş, A.; İkizler, A.A. Model. Meas. Cont. C. 1998, 57, 5963.

10. Kahveci, B.; İkizler, A.A. Acta Polon. Pharm-Drug Res. 2000, 57, 119-122.

11. Kahveci, B.; İkizler, A.A. Turk. J. Chem. 2000, 24, 343-351.

12. Kahveci, B.; Bekircan, O.; Serdar, M.; İkizler, A.A. Indian J. Chem. Sec-B. 2003, 42B, 15271530.

13. Kahveci, B.; Bekircan, O.; Serdar, M.; İkizler, A.A. Rev. Roum. Chim. 2003, 48, 615-618. 
14. Çoruh, U.; Kahveci, B.; Şaşmaz, S.; Ağar, Erbil.; Kim, Y.; Erdönmez, A. Acta Cryst. 2003, C59, o476-0478.

15. Parrick, J.; Yahya, A.; Jin, Y. Tetrahedron Lett. 1984, 25, 3099-3100.

16. Rajapadhye, M.; Popp, F.D. J. Heterocycl. Chem. 1984, 21, 289-291.

17. Morley, J.E.; Farr, S.A.; Flood, J.F. Eur. J. Pharmacol. 1996, 305, 23-29.

18. Bergman, J.; Stalhandske, C.; Vallberg, H. Acta Chem. Scand. 1997, 51, 753-759.

19. Panova, N.G.; Zemskova, A.E. Neurosci. Lett. 1997, 233, 58-60.

20. Pandeya, S.N; Sriram, D. Acta Pharm. Turcica 1998, XXXX, 33-38.

21. Pandeya, S.N.; Sriram, D.; Nath, G.; de Clercq, E. Pharm. Acta Helv. 1999, 74, 11-17.

22. Pandeya, S.N.; Sriram, D.; Nath, G.; de Clercq, E. Eur. J. Med. Chem. 2000, 35, 249-255.

23. Sridhar, S.K.; Saravanan, M.; Ramesh, A. Eur. J. Med. Chem. 2001, 36, 615-625.

24. Pandeya, S.N.; Sriram, D.; Nath, G.; de Clercq, E. Arzneim.-Forsch./Drug Res . 2000, 50, 55-59.

25. Pinner, A. Die Imidoäther und ihre Derivate, 1 Auflage; Oppenheim: Berlin, 1892.

Sample Availability: Available from the author.

(C) 2005 by MDPI (http://www.mdpi.org). Reproduction is permitted for noncommercial purposes. 